\title{
Exact Likelihood Ratio Test for the Parameters of the Linear Regression Model with Normal Errors
}

\author{
Martina Chvosteková, Viktor Witkovský \\ Institute of Measurement Science, Slovak Academy of Sciences, Dúbravská cesta 9, 84104 Bratislava, Slovakia \\ E-mail: chvosta@gmail.com, witkovsky@savba.sk
}

\begin{abstract}
In this paper we present an exact likelihood ratio test (LRT) for testing the simple null hypothesis on all parameters of the linear regression model with normally distributed errors. In particular, we consider the simultaneous test for the regression parameters, $\beta$, and the error standard deviation, $\sigma$. The critical values of the LRT are presented for small sample sizes and a small number of explanatory variables for usual significance levels, $\alpha=0.1,0.05$, and 0.01 . The test is directly applicable for construction of the $(1-\alpha)$-confidence region for the parameters $(\beta, \sigma)$ and the simultaneous tolerance intervals for future observations in linear regression models. For comparison, the suggested method for construction of the tolerance factors of the symmetric $(1-\gamma)$-content simultaneous $(1-\alpha)$-tolerance intervals is illustrated by a simple numerical example.
\end{abstract}

Keywords: linear regression model; exact likelihood ratio test; simultaneous tolerance intervals; confidence-set approach.

\section{INTRODUCTION}

I $\mathrm{N}$ THE PAPER we present an exact likelihood ratio test for testing the simple null hypothesis $H_{0}:(\beta, \sigma)=\left(\beta_{0}, \sigma_{0}\right)$ against the alternative $H_{1}:(\beta, \sigma) \neq\left(\beta_{0}, \sigma_{0}\right)$ on the parameters $\beta$ and $\sigma$ of the linear regression model $Y=X \beta+\sigma Z$ with normally distributed errors, $Z \sim N\left(0, I_{n}\right)$.

Although the derivation of the exact distribution of the likelihood ratio test (LRT) statistic under the null hypothesis $H_{0}$ is straightforward, it seems that the result is not available in the standard (statistical) literature on linear regression models nor in the literature on their applications in measurement science and metrology.

The test is directly applicable for construction of the $(1-\alpha)$-confidence region for the parameters $(\beta, \sigma)$ and for construction of the simultaneous tolerance intervals for future observations in linear regression models. The simultaneous tolerance intervals are important for many measurement procedures. The most common application for simultaneous tolerance intervals is the multiple-use calibration problem; see e.g. [7], [6], [1]. The tolerance intervals have been recognized and considered in various settings by many authors, see e.g. [9], [8], [10], [3], [4], [5], [6], and [2]. These simultaneous tolerance intervals are constructed such that with given confidence level $(1-\alpha)$ at least a specified proportion $(1-\gamma)$ of the population is contained in the tolerance interval for all possible values of the predictor variates. All known simultaneous tolerance intervals in regression are conservative in that the actual confidence level exceeds the nominal level $(1-\alpha)$.

Here we present the method for computing the critical values of the LRT as well as the tables of the critical values for selected small sample sizes in the range $n=k+1, \ldots, 100$ with $k$ explanatory variables, $k=1, \ldots, 10$, and usual significance levels $\alpha=0.1,0.05$, and 0.01 .

The suggested method for construction of the simultane- ous tolerance intervals and/or the tolerance factors of the symmetric $(1-\gamma)$-content simultaneous $(1-\alpha)$-tolerance intervals is illustrated by a simple numerical example.

\section{LIKELIHOOD RATIO TEST OF THE HYPOTHESIS

$$
H_{0}:(\beta, \sigma)=\left(\beta_{0}, \sigma_{0}\right)
$$

Consider the linear regression model $Y=X \beta+\sigma Z$ with normally distributed errors, where $Y$ represents the $n$-dimensional random vector of response variables, $X$ is the $n \times k$ matrix of non-stochastic explanatory variables (for simplicity, here we assume that $X$ is a full-rank matrix), $\beta$ is a $k$-dimensional vector of regression parameters, $Z$ is an $n$-dimensional vector of standard normal errors, i.e. $Z \sim N\left(0, I_{n}\right)$, and $\sigma$ is the error standard deviation, $\sigma>0$.

Here we consider the LRT for testing the simple null hypothesis $H_{0}:(\beta, \sigma)=\left(\beta_{0}, \sigma_{0}\right)$ against the alternative $H_{1}:(\beta, \sigma) \neq\left(\beta_{0}, \sigma_{0}\right)$. Based on the above assumptions the $\log$-likelihood function, denoted as $\ell(\beta, \sigma \mid Y=y, X)$, is given by

$$
\begin{aligned}
\ell(\beta, \sigma \mid y, X)= & -\frac{n}{2} \log (2 \pi)-\frac{n}{2} \log \left(\sigma^{2}\right) \\
& -\frac{1}{2 \sigma^{2}}(y-X \beta)^{\prime}(y-X \beta) .
\end{aligned}
$$

The (-2)-multiple of the logarithm of the LRT statistic, say $\lambda(y \mid X)$ for observed value $y$ of $Y$, and given $X$, for testing the null hypothesis $H_{0}:(\beta, \sigma)=\left(\beta_{0}, \sigma_{0}\right)$ is given by

$$
\begin{aligned}
\lambda(y \mid X) & =-2\left(\sup _{(\beta, \sigma) \in H_{0}} \ell(\beta, \sigma \mid y, X)-\sup _{(\beta, \sigma)} \ell(\beta, \sigma \mid y, X)\right) \\
& =-2\left(\ell\left(\beta_{0}, \sigma_{0} \mid y, X\right)-\ell\left(\hat{\beta}_{M L}, \hat{\sigma}_{M L} \mid y, X\right)\right) \\
& =\frac{1}{\sigma_{0}^{2}}\left(y-X \beta_{0}\right)^{\prime}\left(y-X \beta_{0}\right)-n \log \left(\frac{\hat{\sigma}_{M L}^{2}}{\sigma_{0}^{2}}\right)-n,
\end{aligned}
$$


where $\hat{\beta}_{M L}=\hat{\beta}=\left(X^{\prime} X\right)^{-1} X^{\prime} y$ is the standard least squares estimate (LSE) of $\beta$ (which is also the MLE of $\beta$ ) and $\hat{\sigma}_{M L}$ is the maximum likelihood estimate (MLE) of the standard deviation $\sigma$, i.e. $\hat{\sigma}_{M L}=\sqrt{(y-X \hat{\beta})^{\prime}(y-X \hat{\beta}) / n}$. Under given model assumptions, and under the null hypothesis $H_{0}$, it is straightforward to derive the distribution of the test statistic $\lambda(Y \mid X)$ :

$$
\begin{aligned}
\lambda(Y \mid X) \sim & \frac{1}{\sigma_{0}^{2}}\left(Y-X \beta_{0}\right)^{\prime}\left(Y-X \beta_{0}\right) \\
& -n \log \left(\frac{\left(Y-X \beta_{0}\right)^{\prime} M_{X}\left(Y-X \beta_{0}\right)}{n \sigma_{0}^{2}}\right)-n \\
\sim & Z^{\prime} Z-n \log \left(Z^{\prime} M_{X} Z\right)+n(\log (n)-1) \\
\sim & Z^{\prime}\left(P_{X}+M_{X}\right) Z-n \log \left(Z^{\prime} M_{X} Z\right)+n(\log (n)-1) \\
\sim & Q_{k}+Q_{n-k}-n \log \left(Q_{n-k}\right)+n(\log (n)-1)
\end{aligned}
$$

where $P_{X}=X\left(X^{\prime} X\right)^{-1} X^{\prime}, M_{X}=I_{n}-P_{X}, Z \sim N\left(0, I_{n}\right), Q_{k} \sim \chi_{k}^{2}$ and $Q_{n-k} \sim \chi_{n-k}^{2}$ are two independent random variables with chi-square distributions, with $k$ and $n-k$ degrees of freedom, respectively. Note that the distribution of $\lambda(Y \mid X)$ does not depend on the particular form of the regression design matrix $X$ but only on the number of observations $n$ and on $k=\operatorname{rank}(X)$ - the rank of the matrix $X$, i.e. $\lambda(Y \mid X) \sim \lambda(Y \mid n, k)$.

This LRT rejects the null hypothesis $H_{0}:(\beta, \sigma)=\left(\beta_{0}, \sigma_{0}\right)$ for large values of the observed test statistic $\lambda(y \mid X)$, i.e. for the given significance level $\alpha \in(0,1)$ the test rejects the null hypothesis if

$$
\lambda(y \mid X)>\lambda_{1-\alpha},
$$

where $\lambda_{1-\alpha}$ is the $(1-\alpha)$-quantile of the distribution of the random variable $\lambda(Y \mid n, k)$, given in (3). The quantiles $\lambda_{1-\alpha}$ could be evaluated numerically, by inverting the cumulative distribution function, of the random variable $\lambda(Y \mid n, k)$, denoted by $\mathcal{F}_{L R}(\cdot)$ :

$$
\begin{aligned}
\mathcal{F}_{L R}(x)= & \operatorname{Pr}(\lambda(Y \mid n, k) \leq x) \\
= & \operatorname{Pr}\left(Q_{k} \leq x-Q_{n-k}+n \log \left(Q_{n-k}\right)-n(\log (n)-1)\right) \\
= & \int_{0}^{\infty} \mathcal{F}_{\chi_{k}^{2}}\left(x-q_{n-k}+n \log \left(q_{n-k}\right)-n(\log (n)-1)\right) \\
& \quad \times f_{\chi_{n-k}^{2}}\left(q_{n-k}\right) \mathrm{d} q_{n-k},
\end{aligned}
$$

where $\mathcal{F}_{\chi_{k}^{2}}(\cdot)$ denotes the cumulative distribution function of the chi-square distribution with $k$ degrees of freedom, and $f_{\chi_{n-k}^{2}}(\cdot)$ denotes the probability density function of the chisquare distribution with $n-k$ degrees of freedom.

Notice that since the family of normal distributions meets the regularity conditions, from the standard asymptotic result about the distribution of the LRT $\lambda(Y \mid X)$ we get that $\lambda_{1-\alpha} \rightarrow \chi_{k+1,1-\alpha}^{2}$ as $n \rightarrow \infty$, where $\chi_{k+1,1-\alpha}^{2}$ denotes the $(1-\alpha)$ quantile of chi-square distribution with $k+1$ degrees of freedom.

The critical values of the LRT are presented in the enclosed tables for different number of explanatory variables, $k=1, \ldots, 10$, selected small sample sizes, $n=k+1:(1): 40$, $n=45:(5): 100$ and $\infty$, and for the usual significance levels $\alpha=0.1$ (Table 2), $\alpha=0.05$ (Table 3), and $\alpha=0.01$ (Table 4).

Alternatively, the test of the hypothesis $H_{0}:(\beta, \sigma)=$ $\left(\beta_{0}, \sigma_{0}\right)$ could be based on the test statistic $F^{\star}$ defined as $F^{\star}=\lambda(Y \mid X) /\left(k S^{2} / \sigma_{0}^{2}\right)$, where $S^{2}=(Y-X \hat{\beta})^{\prime}(Y-X \hat{\beta}) /(n-k)$ and $\hat{\beta}=\left(X^{\prime} X\right)^{-1} X^{\prime} Y$. Hence, we get

$$
\begin{aligned}
F^{\star}= & \frac{1}{k} \frac{\left(\hat{\beta}-\beta_{0}\right)^{\prime} X^{\prime} X\left(\hat{\beta}-\beta_{0}\right)}{S^{2}}+\frac{n-k}{k} \\
& -\frac{n}{k} \frac{\log \left((n-k) S^{2} / n \sigma_{0}^{2}\right)+1}{S^{2} / \sigma_{0}^{2}}
\end{aligned}
$$

Note that the leading term in $F^{\star}$ is the standard $F$-statistic for testing the hypothesis on regression parameters $H_{0}: \beta=\beta_{0}$ against the alternative $H_{1}: \beta \neq \beta_{0}$. Under null hypothesis $H_{0}:(\beta, \sigma)=\left(\beta_{0}, \sigma_{0}\right)$ we get directly

$$
F^{\star} \sim \frac{Q_{k} / k}{Q_{n-k} / n-k}+\frac{n-k}{k}-\frac{n}{k} \frac{\log \left(Q_{n-k} / n\right)+1}{Q_{n-k} / n-k} .
$$

Then, the test rejects the null hypothesis if

$$
F_{o b s}^{\star}>F_{1-\alpha}^{\star},
$$

where $F_{o b s}^{\star}$ denotes the observed value of the statistic $F^{\star}$ and $F_{1-\alpha}^{\star}$ is the $(1-\alpha)$-quantile of the distribution of the random variable $F^{\star}$. Similarly as before, the quantiles $F_{1-\alpha}^{\star}$ could be evaluated numerically, by inverting the cumulative distribution function of the random variable $F^{\star}$, denoted by $\mathcal{F}_{F^{\star}}(x)$ :

$$
\begin{aligned}
\mathcal{F}_{F^{\star}}(x)= & \operatorname{Pr}\left(F^{\star} \leq x\right) \\
= & \operatorname{Pr}\left(Q_{k} \leq \frac{x k Q_{n-k}}{n-k}-Q_{n-k}+n\left(\log \left(\frac{Q_{n-k}}{n}\right)+1\right)\right) \\
= & \int_{0}^{\infty} \mathcal{F}_{\chi_{k}^{2}}\left(\frac{x k q_{n-k}}{n-k}-q_{n-k}+n\left(\log \left(\frac{q_{n-k}}{n}\right)+1\right)\right) \\
& \quad \times f_{\chi_{n-k}^{2}}\left(q_{n-k}\right) \mathrm{d} q_{n-k} .
\end{aligned}
$$

\section{Numerical Algorithm}

The Matlab algorithm for computing the quantiles $\lambda_{1-\alpha}$ and $F_{1-\alpha}^{\star}$ is available upon request from the authors.

For those interested in the idea of the algorithm, we present here a simplified working version of the Matlab code used for calculation of the quantiles of the distribution of the random variable $\lambda(Y \mid n, k)$, which is based on the following functions available in Matlab:

FZERO Single-variable nonlinear zero finding. $\mathrm{X}=$ FZERO (FUN , XO) tries to find a zero of the function FUN near $\mathrm{XO}$, if $\mathrm{XO}$ is a scalar.

QUADGK Numerically evaluate integral by adaptive GaussKronrod quadrature. $Q=\operatorname{QUADGK}(\mathrm{FUN}, \mathrm{A}, \mathrm{B})$ attempts to approximate the integral of scalar-valued function FUN from A to B using high order global adaptive quadrature and default error tolerances. 
CHI2CDF Chi-square cumulative distribution function. $\mathrm{P}=$ $\operatorname{CHI} \operatorname{CDF}(\mathrm{X}, \mathrm{V})$ returns the chi-square cumulative distribution function with $\mathrm{V}$ degrees of freedom at the values in X, (Statistics Toolbox).

CHI2PDF Chi-square probability density function (pdf). $\mathrm{Y}=$ $\operatorname{CHI} \operatorname{PDF}(\mathrm{X}, \mathrm{V})$ returns the chi-square pdf with $\mathrm{V}$ degrees of freedom at the values in $\mathrm{X}$, (Statistics Toolbox).

Then, for example, the 0.95 -quantile $\lambda_{1-0.05}$ of the random variable $\lambda(Y \mid n, k)$, based on the linear regression model with $n=15$ observations and $k=2$ regressors, could be calculated by calling the user defined function LRinv:

> quantile $=\operatorname{LRinv}(2,15,0.95)$

which gives as a result the value

$>$ quantile $=8.6813$

The function LRinv could be defined as follows (note that it is calling other auxiliary functions, given bellow):

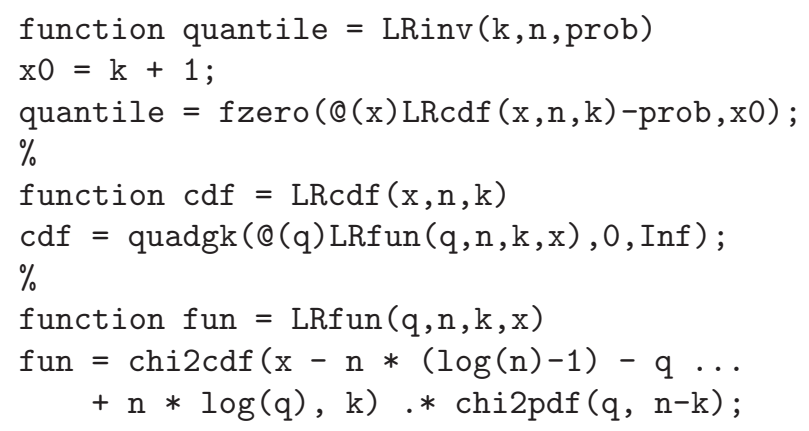

\section{Simultaneous tolerANCE INTERVALS}

The suggested exact tests, (4) and (8), for testing the simple null hypothesis $H_{0}:(\beta, \sigma)=\left(\beta_{0}, \sigma_{0}\right)$, could be directly used to construct the exact simultaneous confidence region for all parameters of the linear regression model. In particular, based on LRT (4), the exact $(1-\alpha)$-confidence region for the parameters $\beta$ and $\sigma$ is given as

$$
C_{1-\alpha}^{L R}(Y \mid X)=\left\{(\beta, \sigma): \lambda(Y \mid X) \leq \lambda_{1-\alpha}\right\} .
$$

Moreover, the confidence regions based on the exact tests could be directly used for constructing the $(1-\gamma)$-content simultaneous $(1-\alpha)$-tolerance intervals in linear regression model with normal errors. These intervals are constructed such that, with confidence $1-\alpha$, we can claim that at least a specified proportion, $1-\gamma$, of the population is contained in the tolerance interval, for all possible values of the predictor variates, see e.g. [1] and [2].

Several authors considered the confidence-set approach for constructing the simultaneous tolerance intervals in linear regression. In [10], Wilson used an ellipsoidal confidence set for the regression coefficients, $\beta$, and standard deviation of the residuals, $\sigma$, which imposes an unnecessary lower bound on $\sigma$, as noted by Limam and Thomas in [5]. They considered two alternative confidence sets. The first is a modification of Wilson's confidence set, which removes the lower bound imposed on $\sigma$, and the second is based on a product set formed from the ellipsoidal confidence set for $\beta$ and a one-sided confidence interval for $\sigma$.

They found the tolerance intervals based on the product confidence set to be efficient and easy to compute compared with those constructed from the ellipsoidal and the modified ellipsoidal confidence sets.

Here we suggest a new procedure for simultaneous $(1-\alpha)$ tolerance intervals covering at least the $(1-\gamma)$-content about the true mean $x^{\prime} \beta$, for any vector $x=\left(x_{1}, \ldots, x_{k}\right)^{\prime}$ of explanatory variables, based on the LRT (4) and the confidence set (10), defined by

$$
\begin{array}{r}
\mathcal{T}_{1-\alpha}^{1-\gamma}(x \mid Y, X)=\left[\inf _{(\beta, \sigma) \in \mathcal{C}_{1-\alpha}(Y \mid X)}\left\{x^{\prime} \beta+u_{\gamma_{1}} \sigma\right\} ;\right. \\
\left.\sup _{(\beta, \sigma) \in C_{1-\alpha}(Y \mid X)}\left\{x^{\prime} \beta+u_{1-\gamma_{2}} \sigma\right\}\right],
\end{array}
$$

where $u_{\gamma_{1}}$ and $u_{1-\gamma_{2}}$ are the pre-specified quantiles of the standard normal distribution, and such that $\gamma=\gamma_{1}+\gamma_{2}$, with $\gamma \in(0,1)$.

As a special case we get the symmetric tolerance intervals about the fitted regression function, with $\gamma_{1}=\gamma_{2}=\gamma / 2$. Notice, that immediately we get the following probability statement

$$
\begin{aligned}
& \operatorname{Pr}\left(\operatorname{Pr}\left(x^{\prime} \beta+\sigma Z \in \mathcal{T}_{1-\alpha}^{1-\gamma}(x \mid Y, X)\right) \geq 1-\gamma,\right. \\
& \quad \text { for all } x \text { and } Z \sim N(0,1), Z \perp Y) \geq 1-\alpha,
\end{aligned}
$$

where $Z \sim N(0,1)$ is a standard normal random variable stochastically independent of the random vector $Y$. Note that considering the exact $F^{\star}$-test (8) could be more appropriate for constructing the simultaneous tolerance intervals.

Traditionally, the symmetric tolerance intervals are presented in the form

$$
\mathcal{T}_{1-\alpha}^{1-\gamma}(x \mid Y, X)=x^{\prime} \hat{\beta} \mp S K(x \mid 1-\alpha, 1-\gamma, Y, X),
$$

where $K(x \mid 1-\alpha, 1-\gamma, Y, X)$ is the tolerance factor which depends on particular values of predictors $x$, confidence coefficient $1-\alpha$, coverage content $(1-\gamma)$, design matrix $X$, error standard deviation $S=\sqrt{(Y-X \hat{\beta})^{\prime}(Y-X \hat{\beta}) /(n-k)}$, and $\hat{\beta}=\left(X^{\prime} X\right)^{-1} X^{\prime} Y$, i.e.

$$
\begin{aligned}
K(x \mid & 1-\alpha, 1-\gamma, Y, X)= \\
= & \frac{1}{S}\left(x^{\prime} \hat{\beta}-\inf _{(\beta, \sigma) \in C_{1-\alpha}(Y \mid X)}\left\{x^{\prime} \beta+u_{\gamma / 2} \sigma\right\}\right) \\
= & \frac{1}{S}\left(\sup _{(\beta, \sigma) \in C_{1-\alpha}(Y \mid X)}\left\{x^{\prime} \beta+u_{1-\gamma / 2} \sigma\right\}-x^{\prime} \hat{\beta}\right) .
\end{aligned}
$$

\section{Numerical Algorithm}

Derivation of the tolerance bounds (11) requires numerical optimization for given $x, \alpha, \gamma$, the design matrix $X$, and the observed vector $y$ of $Y$. 
A simplified version of the Matlab code used for calculation of the lower tolerance bound, given in (11), as well as the tolerance factor, (14), is based on the function:

FMINCON Minimizes the function FUN subject to the nonlinear constraints defined in NONLCON (Optimization Toolbox).

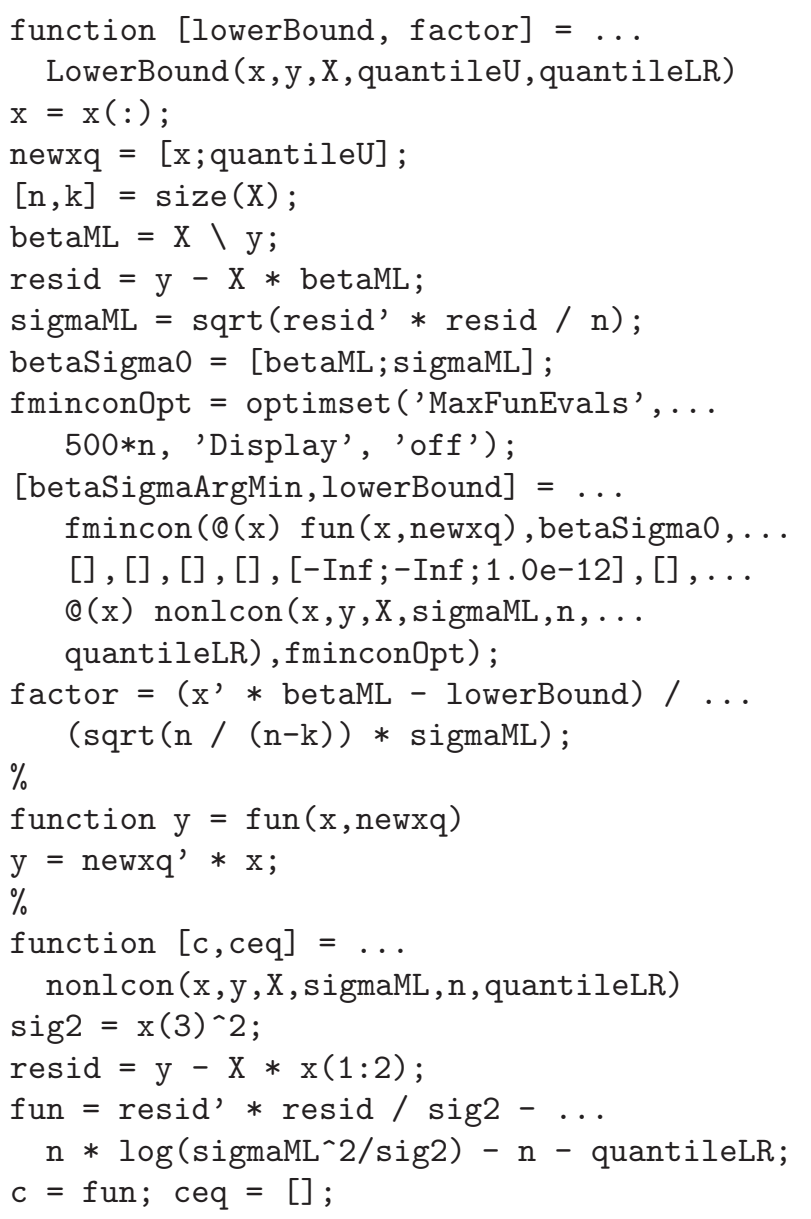

An alternative algorithm for the approximate solution based on Monte Carlo simulations, which is especially useful for linear regression models with $k>2$, was suggested in [11].

\section{EXAMPLE}

For numerical illustration we consider fifteen hypothetical pairs of values $\left(x_{i}, y_{i}\right)$ selected for the speed-orifice problem, as considered and studied in [3] and [5], the measurement values are given in Table 1.

A simple linear regression model $Y_{i}=\beta_{1}+\beta_{2} x_{i}+\sigma Z_{i}$, with independent errors $Z_{i} \sim N(0,1), i=1, \ldots, 15$ (i.e. $n=15$ and $k=2$ ), was considered for the analysis of the speed-orifice measurements. The ML estimates $\hat{\beta}$ of the regression parameter $\beta=\left(\beta_{1}, \beta_{2}\right)^{\prime}$ and $\hat{\sigma}_{M L}$ of the standard deviation $\sigma$ are

$$
\hat{\beta}=(-19041.86,17929.64)^{\prime}, \quad \hat{\sigma}_{M L}=121.50 .
$$

Moreover, $\bar{x}=\frac{1}{n} \sum_{i=1}^{n} x_{i}=1.3531, S_{x}=\frac{1}{n-1} \sum_{i=1}^{n}\left(x_{i}-\bar{x}\right)^{2}=$ 0.0292 .

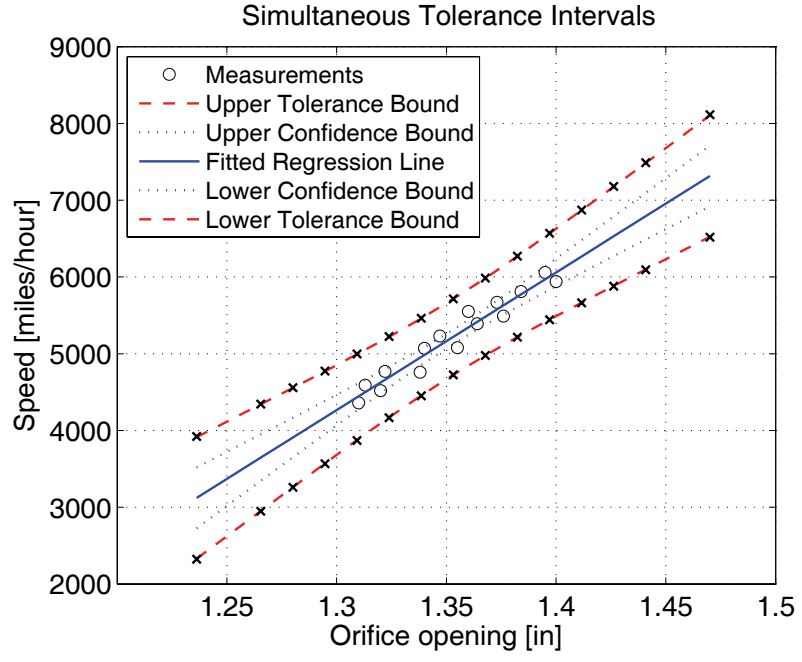

Figure 1. The $(1-\gamma)$-content symmetric simultaneous $(1-\alpha)$ tolerance intervals for the speed-orifice problem calculated at several specified predictors at the significance levels $\alpha=0.05$ and for prespecified population content $1-\gamma$, with $\gamma=0.05$. The data and the explicit values of the tolerance factors for the selected simultaneous tolerance bounds, depicted by the symbol $\times$, are presented in Table 1 .

For given $k=2, n=15$, and the significance levels $\alpha=$ $0.05,0.01$, the critical values of the test $(4)$ and $(8)$, respectively, for testing the null hypothesis $H_{0}:(\beta, \sigma)=\left(\beta_{0}, \sigma_{0}\right)$, are

$$
\begin{array}{ll}
\lambda_{1-0.05}=8.6813, & \lambda_{1-0.01}=12.6160, \\
F_{1-0.05}^{*}=8.1578, & F_{1-0.01}^{*}=17.3985 .
\end{array}
$$

Compare with the Table 3 and Table 4 . The tables of critical values $F_{1-\alpha}^{\star}$ are not presented in this paper.

The tolerance factors of the $(1-\gamma)$-content symmetric simultaneous $(1-\alpha)$-tolerance intervals for the speed-orifice problem calculated at several specified predictors $f_{i}, i=$ $1, \ldots, 15$, (see the Table 1, columns 3-4, respectively), for different combinations of selected significance levels $\alpha=0.05$, $\alpha=0.01$, and population content $1-\gamma$, for $\gamma=0.05$ and $\gamma=0.01$, are given in Table 1, (see the columns 5-8).

For illustration, using the function LowerBound for the speed-orifice data with the predictor vector $x=(1,1.3531)$, with the quantile of standard normal distribution $u_{\gamma / 2}=-1.96$ for selected significance levels $\gamma=0.05$ and with the quantile $\lambda_{1-\alpha}=8.6813$ for selected confidence coefficient $1-\alpha=0.05$, we get

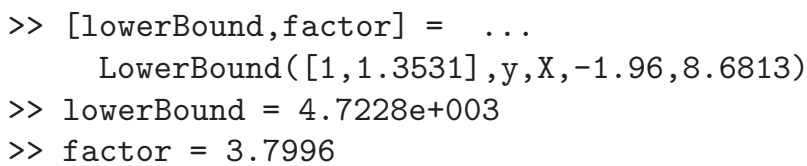

The upper tolerance bound could be calculated from the tolerance factor, see (13), or directly by using the function LowerBound - by minimizing the function $-\left(x^{\prime} \beta+u_{1-\gamma / 2}\right)$. 
Table 1: Tolerance factors of the $(1-\gamma)$-content symmetric simultaneous $(1-\alpha)$-tolerance intervals for the speed-orifice problem calculated at several specified predictors for different combinations of selected significance levels $\alpha=0.05, \alpha=0.01$, and population content $1-\gamma$, for $\gamma=0.05$ and $\gamma=0.01$.

\begin{tabular}{|c|c|c|c|c|c|c|c|}
\hline \multirow{3}{*}{$\begin{array}{c}\text { Speed } \\
\text { [miles/hour] } \\
y_{i}\end{array}$} & \multirow{3}{*}{$\begin{array}{c}\text { Orifice Opening } \\
\text { [in] } \\
x_{i}\end{array}$} & \multirow{3}{*}{$\begin{array}{c}\text { Predictors } \\
\text { [in] } \\
f_{i}\end{array}$} & \multirow{3}{*}{$\begin{array}{l}\text { Standardized } \\
\text { Predictors } \\
\left(f_{i}-\bar{x}\right) / S_{x}\end{array}$} & \multicolumn{4}{|c|}{ Tolerance Factors } \\
\hline & & & & \multicolumn{2}{|c|}{$\alpha=0.05$} & \multicolumn{2}{|c|}{$\alpha=0.01$} \\
\hline & & & & $\gamma=0.05$ & $\gamma=0.01$ & $\gamma=0.05$ & $\gamma=0.01$ \\
\hline 4360 & 1.3100 & 1.2362 & -4 & 6.1212 & 7.0053 & 7.5563 & 8.5817 \\
\hline 4590 & 1.3130 & 1.2654 & -3 & 5.3466 & 6.2590 & 6.5510 & 7.6125 \\
\hline 4520 & 1.3200 & 1.2800 & -2.5 & 4.9779 & 5.9090 & 6.0722 & 7.1578 \\
\hline 4770 & 1.3220 & 1.2947 & -2 & 4.6298 & 5.5836 & 5.6201 & 6.7348 \\
\hline 4760 & 1.3380 & 1.3093 & -1.5 & 4.3139 & 5.2946 & 5.2095 & 6.3585 \\
\hline 5070 & 1.3400 & 1.3239 & -1 & 4.0495 & 5.0593 & 4.8654 & 6.0519 \\
\hline 5230 & 1.3470 & 1.3385 & -0.5 & 3.8664 & 4.9014 & 4.6268 & 5.8459 \\
\hline 5080 & 1.3550 & 1.3531 & 0 & 3.7996 & 4.8451 & 4.5396 & 5.7723 \\
\hline 5550 & 1.3600 & 1.3678 & 0.5 & 3.8664 & 4.9014 & 4.6268 & 5.8459 \\
\hline 5390 & 1.3640 & 1.3824 & 1 & 4.0495 & 5.0593 & 4.8654 & 6.0519 \\
\hline 5670 & 1.3730 & 1.3970 & 1.5 & 4.3139 & 5.2946 & 5.2095 & 6.3585 \\
\hline 5490 & 1.3760 & 1.4116 & 2 & 4.6298 & 5.5836 & 5.6201 & 6.7348 \\
\hline 5810 & 1.3840 & 1.4262 & 2.5 & 4.9779 & 5.9090 & 6.0722 & 7.1578 \\
\hline 6060 & 1.3950 & 1.4408 & 3 & 5.3466 & 6.2590 & 6.5510 & 7.6125 \\
\hline 5940 & 1.4000 & 1.4701 & 4 & 6.1212 & 7.0053 & 7.5563 & 8.5817 \\
\hline
\end{tabular}

\section{Discussion}

The presented exact likelihood ratio test (4) for testing the null hypothesis $H_{0}:(\beta, \sigma)=\left(\beta_{0}, \sigma_{0}\right)$ against the alternative $H_{1}:(\beta, \sigma) \neq\left(\beta_{0}, \sigma_{0}\right)$ on the parameters $\beta$ and $\sigma$ of the linear regression model $Y=X \beta+\sigma Z$ with normally distributed errors, $Z \sim N\left(0, I_{n}\right)$, is especially useful for construction of the simultaneous confidence region for the regression parameters, see (10), and for the simultaneous tolerance intervals (11), and/or the tolerance factors (14), respectively, for future observations predicted for any value of the predictor vector $x$.

If compared with the $(1-\gamma)$-content symmetric simultaneous $(1-\alpha)$-tolerance intervals based on the product-set approach, as proposed by Limam and Thomas in [5], the suggested tolerance intervals given by (11) are slightly broader for the predictor values close to the middle of the fitted regression function, and became narrower for more distant predictors.

\section{ACKNOWLEDGMENTS}

The research was supported by the grants VEGA 1/0077/09, VEGA 2/7087/27, APVV-SK-AT-0003-09, and APVVRPEU-0008-06.

\section{REFERENCES}

[1] De Gryze, S., Langhans, I. \& Vandebroek, M. (2007). Using the correct intervals for prediction: A tutorial on tolerance intervals for ordinary least-squares regression. Chemometrics and Intelligent Laboratory Systems 87, 147-154.

[2] Krishnamoorthy, K. \& Mathew, T. (2009). Statistical Tolerance Regions: Theory, Applications, and Computation. Wiley.

[3] Lieberman, G. J. \& Miller, R. G. Jr. (1963). Simultaneous tolerance intervals in regression. Biometrika 50, 155-168.

[4] Lieberman, G. J., Miller, R. G. Jr. \& Hamilton, A. (1967). Unlimited simultaneous discrimination intervals in regression. Biometrika 54, 133-145. Corrections in Biometrika 58, 687.

[5] Limam, M. M. T. \& Thomas, D. R. (1988). Simultaneous tolerance intervals for the linear regression model. Journal of the American Statistical Association 83 (403), 801-804.

[6] Mee R. W., Eberhardt, K. R. \& Reeve, C. P. (1991). Calibration and simultaneous tolerance intervals for regression. Technometrics 33 (2), 211-219.

[7] Scheffé, H. (1973). A statistical theory of calibration. Annals of Statistics 1(1), 1-37.

[8] Wallis, W. A. (1951). Tolerance intervals for linear regression. In Proceedings of the Second Berkeley Symposium on Mathematical Statistics and Probability, Berkeley CA: University of California Press, Berkeley.

[9] Wilks, S. S. (1942). Statistical prediction with special reference to the problem of tolerance limits. The Annals of Mathematical Statistics 13, 400-409.

[10] Wilson, A. L. (1967). An approach to simultaneous tolerance intervals in regression. The Annals of Mathematical Statistics 38(5), 1536-1540.

[11] Witkovský, V. \& Chvosteková M. (2009). Simultaneous tolerance intervals for the linear regression model. In MEASUREMENT 2009. Proceedings of the International Conference on Measurement, Smolenice, May 20-23, 2009. 
Table 2: Critical values of the likelihood ratio test (LRT) for testing the null hypothesis $H_{0}:(\beta, \sigma)=\left(\beta_{0}, \sigma_{0}\right)$ against the alternative $H_{1}:(\beta, \sigma) \neq\left(\beta_{0}, \sigma_{0}\right)$ on parameters of the normal linear regression model with $k, k=1, \ldots, 10$, explanatory variables, selected small sample sizes $n, n=k+1, \ldots, 100$, and the significance level $\alpha=0.1$.

\begin{tabular}{|c|c|c|c|c|c|c|c|c|c|c|}
\hline $\mathbf{n} / \mathbf{k}$ & 1 & 2 & 3 & 4 & 5 & 6 & 7 & 8 & 9 & 10 \\
\hline 2 & 9.0856 & - & - & - & - & - & - & - & - & - \\
\hline 3 & 6.8029 & 15.1840 & - & - & - & - & - & - & - & - \\
\hline 4 & 6.0549 & 10.6953 & 21.6002 & - & - & - & - & - & - & - \\
\hline 5 & 5.6856 & 9.2154 & 14.7996 & 28.2608 & - & - & - & - & - & - \\
\hline 6 & 5.4659 & 8.4775 & 12.5243 & 19.0877 & 35.1190 & - & - & - & - & - \\
\hline 7 & 5.3203 & 8.0346 & 11.3749 & 15.9760 & 23.5341 & 42.1428 & - & - & - & - \\
\hline 8 & 5.2168 & 7.7393 & 10.6781 & 14.3856 & 19.5577 & 28.1187 & 49.3085 & - & - & - \\
\hline 9 & 5.1395 & 7.5279 & 10.2095 & 13.4126 & 17.5048 & 23.2566 & 32.8254 & 56.5988 & - & - \\
\hline 10 & 5.0795 & 7.3695 & 9.8722 & 12.7532 & 16.2386 & 20.7249 & 27.0617 & 37.6412 & 64.0000 & - \\
\hline 11 & 5.0316 & 7.2460 & 9.6176 & 12.2757 & 15.3748 & 19.1522 & 24.0382 & 30.9634 & 42.5556 & 71.5009 \\
\hline 12 & 4.9925 & 7.1471 & 9.4185 & 11.9136 & 14.7461 & 18.0733 & 22.1485 & 27.4378 & 34.9535 & 47.5599 \\
\hline 13 & 4.9599 & 7.0662 & 9.2585 & 11.6292 & 14.2670 & 17.2841 & 20.8455 & 25.2223 & 30.9172 & 39.0252 \\
\hline 14 & 4.9324 & 6.9986 & 9.1271 & 11.3998 & 13.8893 & 16.6803 & 19.8882 & 23.6877 & 28.3688 & 34.4711 \\
\hline 15 & 4.9089 & 6.9414 & 9.0171 & 11.2108 & 13.5838 & 16.2027 & 19.1532 & 22.5561 & 26.5963 & 31.5834 \\
\hline 16 & 4.8885 & 6.8924 & 8.9239 & 11.0524 & 13.3313 & 15.8151 & 18.5701 & 21.6843 & 25.2847 & 29.5677 \\
\hline 17 & 4.8707 & 6.8499 & 8.8437 & 10.9176 & 13.1190 & 15.4940 & 18.0955 & 20.9906 & 24.2713 & 28.0715 \\
\hline 18 & 4.8550 & 6.8126 & 8.7740 & 10.8015 & 12.9381 & 15.2236 & 17.7014 & 20.4246 & 23.4629 & 26.9123 \\
\hline 19 & 4.8410 & 6.7798 & 8.7129 & 10.7005 & 12.7820 & 14.9925 & 17.3687 & 19.9537 & 22.8018 & 25.9853 \\
\hline 20 & 4.8286 & 6.7505 & 8.6589 & 10.6117 & 12.6459 & 14.7928 & 17.0839 & 19.5553 & 22.2505 & 25.2257 \\
\hline 21 & 4.8173 & 6.7244 & 8.6108 & 10.5332 & 12.5262 & 14.6183 & 16.8374 & 19.2137 & 21.7834 & 24.5911 \\
\hline 22 & 4.8072 & 6.7008 & 8.5678 & 10.4631 & 12.4200 & 14.4647 & 16.6218 & 18.9176 & 21.3823 & 24.0524 \\
\hline 23 & 4.7979 & 6.6795 & 8.5289 & 10.4002 & 12.3253 & 14.3283 & 16.4315 & 18.6581 & 21.0339 & 23.5892 \\
\hline 24 & 4.7895 & 6.6601 & 8.4937 & 10.3435 & 12.2401 & 14.2063 & 16.2625 & 18.4290 & 20.7283 & 23.1863 \\
\hline 25 & 4.7818 & 6.6424 & 8.4617 & 10.2921 & 12.1633 & 14.0967 & 16.1112 & 18.2251 & 20.4581 & 22.8325 \\
\hline 26 & 4.7747 & 6.6262 & 8.4324 & 10.2453 & 12.0935 & 13.9976 & 15.9750 & 18.0424 & 20.2174 & 22.5193 \\
\hline 27 & 4.7682 & 6.6112 & 8.4056 & 10.2024 & 12.0298 & 13.9075 & 15.8517 & 17.8779 & 20.0016 & 22.2400 \\
\hline 28 & 4.7621 & 6.5974 & 8.3808 & 10.1630 & 11.9716 & 13.8253 & 15.7396 & 17.7288 & 19.8069 & 21.9893 \\
\hline 29 & 4.7565 & 6.5847 & 8.3580 & 10.1268 & 11.9180 & 13.7500 & 15.6373 & 17.5930 & 19.6304 & 21.7630 \\
\hline 30 & 4.7513 & 6.5728 & 8.3368 & 10.0932 & 11.8686 & 13.6807 & 15.5434 & 17.4690 & 19.4696 & 21.5576 \\
\hline 31 & 4.7464 & 6.5617 & 8.3171 & 10.0621 & 11.8229 & 13.6168 & 15.4569 & 17.3552 & 19.3225 & 21.3704 \\
\hline 32 & 4.7418 & 6.5514 & 8.2987 & 10.0332 & 11.7805 & 13.5576 & 15.3771 & 17.2503 & 19.1874 & 21.1990 \\
\hline 33 & 4.7375 & 6.5418 & 8.2816 & 10.0062 & 11.7411 & 13.5026 & 15.3032 & 17.1534 & 19.0629 & 21.0415 \\
\hline 34 & 4.7335 & 6.5327 & 8.2656 & 9.9810 & 11.7043 & 13.4514 & 15.2345 & 17.0636 & 18.9478 & 20.8962 \\
\hline 35 & 4.7297 & 6.5242 & 8.2505 & 9.9574 & 11.6699 & 13.4037 & 15.1706 & 16.9801 & 18.8411 & 20.7618 \\
\hline 36 & 4.7261 & 6.5162 & 8.2364 & 9.9353 & 11.6376 & 13.3590 & 15.1108 & 16.9023 & 18.7418 & 20.6371 \\
\hline 37 & 4.7228 & 6.5087 & 8.2231 & 9.9145 & 11.6074 & 13.3172 & 15.0549 & 16.8296 & 18.6492 & 20.5211 \\
\hline 38 & 4.7196 & 6.5015 & 8.2105 & 9.8948 & 11.5789 & 13.2778 & 15.0025 & 16.7616 & 18.5627 & 20.4129 \\
\hline 39 & 4.7166 & 6.4948 & 8.1987 & 9.8763 & 11.5521 & 13.2408 & 14.9532 & 16.6977 & 18.4816 & 20.3117 \\
\hline 40 & 4.7137 & 6.4884 & 8.1874 & 9.8588 & 11.5267 & 13.2059 & 14.9068 & 16.6377 & 18.4056 & 20.2168 \\
\hline 45 & 4.7013 & 6.4609 & 8.1392 & 9.7840 & 11.4187 & 13.0576 & 14.7104 & 16.3844 & 18.0857 & 19.8197 \\
\hline 50 & 4.6915 & 6.4391 & 8.1012 & 9.7252 & 11.3343 & 12.9422 & 14.5583 & 16.1893 & 17.8408 & 19.5174 \\
\hline 55 & 4.6835 & 6.4214 & 8.0705 & 9.6778 & 11.2664 & 12.8499 & 14.4371 & 16.0345 & 17.6472 & 19.2795 \\
\hline 60 & 4.6771 & 6.4068 & 8.0451 & 9.6388 & 11.2108 & 12.7743 & 14.3382 & 15.9086 & 17.4903 & 19.0873 \\
\hline 65 & 4.6712 & 6.3945 & 8.0238 & 9.6061 & 11.1643 & 12.7113 & 14.2559 & 15.8041 & 17.3605 & 18.9288 \\
\hline 70 & 4.6664 & 6.3840 & 8.0056 & 9.5784 & 11.1248 & 12.6580 & 14.1865 & 15.7161 & 17.2514 & 18.7958 \\
\hline 75 & 4.6623 & 6.3749 & 7.9900 & 9.5545 & 11.0909 & 12.6123 & 14.1271 & 15.6410 & 17.1584 & 18.6827 \\
\hline 80 & 4.6587 & 6.3670 & 7.9764 & 9.5337 & 11.0615 & 12.5727 & 14.0756 & 15.5760 & 17.0781 & 18.5852 \\
\hline 85 & 4.6555 & 6.3600 & 7.9644 & 9.5155 & 11.0357 & 12.5381 & 14.0307 & 15.5193 & 17.0081 & 18.5004 \\
\hline 90 & 4.6527 & 6.3539 & 7.9538 & 9.4994 & 11.0130 & 12.5075 & 13.9910 & 15.4694 & 16.9466 & 18.4259 \\
\hline 95 & 4.6502 & 6.3484 & 7.9444 & 9.4850 & 10.9927 & 12.4803 & 13.9558 & 15.4251 & 16.8921 & 18.3599 \\
\hline 100 & 4.6479 & 6.3434 & 7.9359 & 9.4722 & 10.9746 & 12.4559 & 13.9244 & 15.3855 & 16.8434 & 18.3012 \\
\hline$\infty$ & 4.6052 & 6.2514 & 7.7794 & 9.2364 & 10.6446 & 12.0170 & 13.3616 & 14.6837 & 15.9872 & 17.2750 \\
\hline
\end{tabular}


Table 3: Critical values of the likelihood ratio test (LRT) for testing the null hypothesis $H_{0}:(\beta, \sigma)=\left(\beta_{0}, \sigma_{0}\right)$ against the alternative $H_{1}:(\beta, \sigma) \neq\left(\beta_{0}, \sigma_{0}\right)$ on parameters of the normal linear regression model with $k, k=1, \ldots, 10$, explanatory variables, selected small sample sizes $n, n=k+1, \ldots, 100$, and the significance level $\alpha=0.05$.

\begin{tabular}{|c|c|c|c|c|c|c|c|c|c|c|}
\hline $\mathbf{n} / \mathbf{k}$ & 1 & 2 & 3 & 4 & 5 & 6 & 7 & 8 & 9 & 10 \\
\hline 2 & 11.8545 & - & - & - & - & - & - & - & - & - \\
\hline 3 & 8.8706 & 19.3470 & - & - & - & - & - & - & - & - \\
\hline 4 & 7.8893 & 13.4989 & 27.1540 & - & - & - & - & - & - & - \\
\hline 5 & 7.4046 & 11.5844 & 18.3296 & 35.2052 & - & - & - & - & - & - \\
\hline 6 & 7.1164 & 10.6358 & 15.4138 & 23.3410 & 43.4538 & - & - & - & - & - \\
\hline 7 & 6.9257 & 10.0694 & 13.9556 & 19.3806 & 28.5094 & 51.8679 & - & - & - & - \\
\hline 8 & 6.7901 & 9.6927 & 13.0778 & 17.3814 & 23.4748 & 33.8153 & 60.4240 & - & - & - \\
\hline 9 & 6.6888 & 9.4241 & 12.4904 & 16.1687 & 20.9120 & 27.6847 & 39.2429 & 69.1047 & - & - \\
\hline 10 & 6.6103 & 9.2228 & 12.0691 & 15.3518 & 19.3464 & 24.5412 & 31.9998 & 44.7793 & 77.8962 & - \\
\hline 11 & 6.5477 & 9.0663 & 11.7521 & 14.7630 & 18.2858 & 22.6089 & 28.2622 & 36.4110 & 50.4142 & 86.7873 \\
\hline 12 & 6.4966 & 8.9412 & 11.5047 & 14.3179 & 17.5176 & 21.2931 & 25.9522 & 32.0684 & 40.9101 & 56.1388 \\
\hline 13 & 6.4541 & 8.8388 & 11.3063 & 13.9693 & 16.9345 & 20.3360 & 24.3719 & 29.3717 & 35.9540 & 45.4906 \\
\hline 14 & 6.4182 & 8.7536 & 11.1435 & 13.6888 & 16.4763 & 19.6068 & 23.2179 & 27.5192 & 32.8629 & 39.9134 \\
\hline 15 & 6.3874 & 8.6813 & 11.0075 & 13.4581 & 16.1065 & 19.0319 & 22.3357 & 26.1616 & 30.7317 & 36.4216 \\
\hline 16 & 6.3608 & 8.6195 & 10.8923 & 13.2649 & 15.8015 & 18.5667 & 21.6383 & 25.1207 & 29.1648 & 34.0061 \\
\hline 17 & 6.3375 & 8.5659 & 10.7933 & 13.1008 & 15.5456 & 18.1821 & 21.0724 & 24.2955 & 27.9601 & 32.2250 \\
\hline 18 & 6.3170 & 8.5190 & 10.7074 & 12.9596 & 15.3278 & 17.8587 & 20.6035 & 23.6244 & 27.0028 & 30.8522 \\
\hline 19 & 6.2988 & 8.4776 & 10.6321 & 12.8369 & 15.1400 & 17.5829 & 20.2085 & 23.0673 & 26.2225 & 29.7589 \\
\hline 20 & 6.2825 & 8.4408 & 10.5656 & 12.7292 & 14.9765 & 17.3448 & 19.8711 & 22.5971 & 25.5736 & 28.8659 \\
\hline 21 & 6.2679 & 8.4079 & 10.5064 & 12.6339 & 14.8329 & 17.1371 & 19.5793 & 22.1946 & 25.0249 & 28.1220 \\
\hline 22 & 6.2546 & 8.3783 & 10.4533 & 12.5490 & 14.7056 & 16.9544 & 19.3244 & 21.8462 & 24.5546 & 27.4919 \\
\hline 23 & 6.2426 & 8.3515 & 10.4056 & 12.4728 & 14.5920 & 16.7923 & 19.0998 & 21.5414 & 24.1468 & 26.9512 \\
\hline 24 & 6.2316 & 8.3271 & 10.3623 & 12.4041 & 14.4901 & 16.6475 & 18.9004 & 21.2725 & 23.7897 & 26.4816 \\
\hline 25 & 6.2216 & 8.3048 & 10.3230 & 12.3419 & 14.3981 & 16.5175 & 18.7221 & 21.0335 & 23.4743 & 26.0700 \\
\hline 26 & 6.2123 & 8.2844 & 10.2870 & 12.2852 & 14.3146 & 16.3999 & 18.5618 & 20.8196 & 23.1936 & 25.7060 \\
\hline 27 & 6.2038 & 8.2657 & 10.2540 & 12.2334 & 14.2386 & 16.2932 & 18.4167 & 20.6270 & 22.9421 & 25.3817 \\
\hline 28 & 6.1959 & 8.2483 & 10.2236 & 12.1858 & 14.1689 & 16.1959 & 18.2849 & 20.4526 & 22.7155 & 25.0910 \\
\hline 29 & 6.1885 & 8.2323 & 10.1955 & 12.1420 & 14.1050 & 16.1067 & 18.1646 & 20.2941 & 22.5102 & 24.8288 \\
\hline 30 & 6.1817 & 8.2174 & 10.1695 & 12.1014 & 14.0460 & 16.0247 & 18.0543 & 20.1492 & 22.3234 & 24.5911 \\
\hline 31 & 6.1753 & 8.2035 & 10.1454 & 12.0639 & 13.9915 & 15.9491 & 17.9528 & 20.0163 & 22.1525 & 24.3745 \\
\hline 32 & 6.1694 & 8.1906 & 10.1229 & 12.0290 & 13.9409 & 15.8791 & 17.8592 & 19.8940 & 21.9958 & 24.1764 \\
\hline 33 & 6.1638 & 8.1785 & 10.1019 & 11.9964 & 13.8938 & 15.8142 & 17.7725 & 19.7811 & 21.8513 & 23.9944 \\
\hline 34 & 6.1586 & 8.1671 & 10.0822 & 11.9660 & 13.8500 & 15.7538 & 17.6919 & 19.6764 & 21.7179 & 23.8267 \\
\hline 35 & 6.1536 & 8.1564 & 10.0638 & 11.9376 & 13.8089 & 15.6974 & 17.6170 & 19.5792 & 21.5941 & 23.6717 \\
\hline 36 & 6.1490 & 8.1464 & 10.0464 & 11.9108 & 13.7705 & 15.6447 & 17.5470 & 19.4886 & 21.4791 & 23.5278 \\
\hline 37 & 6.1446 & 8.1369 & 10.0301 & 11.8857 & 13.7345 & 15.5952 & 17.4815 & 19.4040 & 21.3719 & 23.3941 \\
\hline 38 & 6.1404 & 8.1280 & 10.0147 & 11.8621 & 13.7005 & 15.5488 & 17.4201 & 19.3248 & 21.2718 & 23.2693 \\
\hline 39 & 6.1363 & 8.1195 & 10.0002 & 11.8398 & 13.6686 & 15.5052 & 17.3624 & 19.2505 & 21.1780 & 23.1528 \\
\hline 40 & 6.1328 & 8.1115 & 9.9864 & 11.8187 & 13.6384 & 15.4640 & 17.3081 & 19.1807 & 21.0900 & 23.0435 \\
\hline 45 & 6.1167 & 8.0770 & 9.9274 & 11.7285 & 13.5098 & 15.2892 & 17.0783 & 18.8863 & 20.7204 & 22.5867 \\
\hline 50 & 6.1038 & 8.0497 & 9.8809 & 11.6577 & 13.4094 & 15.1533 & 16.9006 & 18.6599 & 20.4377 & 22.2394 \\
\hline 55 & 6.0934 & 8.0276 & 9.8433 & 11.6007 & 13.3288 & 15.0446 & 16.7591 & 18.4803 & 20.2144 & 21.9663 \\
\hline 60 & 6.0847 & 8.0092 & 9.8122 & 11.5537 & 13.2627 & 14.9557 & 16.6437 & 18.3343 & 20.0335 & 21.7459 \\
\hline 65 & 6.0774 & 7.9938 & 9.7861 & 11.5144 & 13.2074 & 14.8816 & 16.5478 & 18.2134 & 19.8840 & 21.5643 \\
\hline 70 & 6.0712 & 7.9806 & 9.7640 & 11.4811 & 13.1606 & 14.8190 & 16.4668 & 18.1115 & 19.7584 & 21.4120 \\
\hline 75 & 6.0658 & 7.9693 & 9.7448 & 11.4523 & 13.1204 & 14.7653 & 16.3975 & 18.0244 & 19.6513 & 21.2824 \\
\hline 80 & 6.0611 & 7.9594 & 9.7282 & 11.4274 & 13.0855 & 14.7187 & 16.3376 & 17.9493 & 19.5590 & 21.1709 \\
\hline 85 & 6.0570 & 7.9507 & 9.7136 & 11.4055 & 13.0549 & 14.6780 & 16.2852 & 17.8837 & 19.4785 & 21.0738 \\
\hline 90 & 6.0533 & 7.9429 & 9.7006 & 11.3861 & 13.0279 & 14.6421 & 16.2391 & 17.8259 & 19.4078 & 20.9886 \\
\hline 95 & 6.0500 & 7.9360 & 9.6891 & 11.3689 & 13.0038 & 14.6102 & 16.1981 & 17.7747 & 19.3451 & 20.9132 \\
\hline 100 & 6.0470 & 7.9299 & 9.6788 & 11.3534 & 12.9823 & 14.5816 & 16.1615 & 17.7290 & 19.2892 & 20.8460 \\
\hline$\infty$ & 5.9915 & 7.8147 & 9.4877 & 11.0705 & 12.5916 & 14.0671 & 15.5073 & 16.9190 & 18.3070 & 19.6751 \\
\hline
\end{tabular}


Table 4: Critical values of the likelihood ratio test (LRT) for testing the null hypothesis $H_{0}:(\beta, \sigma)=\left(\beta_{0}, \sigma_{0}\right)$ against the alternative $H_{1}:(\beta, \sigma) \neq\left(\beta_{0}, \sigma_{0}\right)$ on parameters of the normal linear regression model with $k, k=1, \ldots, 10$, explanatory variables, selected small sample sizes $n, n=k+1, \ldots, 100$, and the significance level $\alpha=0.01$.

\begin{tabular}{|c|c|c|c|c|c|c|c|c|c|c|}
\hline $\mathbf{n} / \mathbf{k}$ & 1 & 2 & 3 & 4 & 5 & 6 & 7 & 8 & 9 & 10 \\
\hline 2 & 18.2902 & - & - & - & - & - & - & - & - & - \\
\hline 3 & 13.6860 & 29.0049 & - & - & - & - & - & - & - & - \\
\hline 4 & 12.1608 & 19.9602 & 40.0323 & - & - & - & - & - & - & - \\
\hline 5 & 11.4053 & 17.0089 & 26.4262 & 51.3036 & - & - & - & - & - & - \\
\hline 6 & 10.9553 & 15.5571 & 21.9730 & 33.0704 & 62.7725 & - & - & - & - & - \\
\hline 7 & 10.6585 & 14.6967 & 19.7741 & 27.0678 & 39.8704 & 74.4068 & - & - & - & - \\
\hline 8 & 10.4474 & 14.1276 & 18.4648 & 24.0871 & 32.2873 & 46.8075 & 86.1831 & - & - & - \\
\hline 9 & 10.2898 & 13.7236 & 17.5960 & 22.3031 & 28.5000 & 37.6208 & 53.8658 & 98.0839 & - & - \\
\hline 10 & 10.1676 & 13.4219 & 16.9772 & 21.1139 & 26.2217 & 33.0087 & 43.0585 & 61.0328 & 110.0954 & - \\
\hline 11 & 10.0705 & 13.1882 & 16.5139 & 20.2636 & 24.6963 & 30.2212 & 37.6075 & 48.5916 & 68.2983 & 122.2068 \\
\hline 12 & 9.9911 & 13.0017 & 16.1540 & 19.6250 & 23.6016 & 28.3473 & 34.2989 & 42.2904 & 54.2123 & 75.6532 \\
\hline 13 & 9.9249 & 12.8495 & 15.8663 & 19.1275 & 22.7767 & 26.9976 & 32.0664 & 38.4511 & 47.0516 & 59.9139 \\
\hline 14 & 9.8693 & 12.7229 & 15.6308 & 18.7288 & 22.1322 & 25.9775 & 30.4533 & 35.8518 & 42.6737 & 51.8861 \\
\hline 15 & 9.8217 & 12.6160 & 15.4351 & 18.4020 & 21.6145 & 25.1784 & 29.2306 & 33.9680 & 39.7007 & 46.9629 \\
\hline 16 & 9.7804 & 12.5245 & 15.2692 & 18.1293 & 21.1893 & 24.5350 & 28.2704 & 32.5365 & 37.5402 & 43.6102 \\
\hline 17 & 9.7443 & 12.4452 & 15.1271 & 17.8982 & 20.8337 & 24.0055 & 27.4956 & 31.4097 & 35.8945 & 41.1679 \\
\hline 18 & 9.7126 & 12.3760 & 15.0040 & 17.6998 & 20.5319 & 23.5619 & 26.8567 & 30.4986 & 34.5963 & 39.3033 \\
\hline 19 & 9.6845 & 12.3149 & 14.8962 & 17.5276 & 20.2725 & 23.1847 & 26.3205 & 29.7459 & 33.5447 & 37.8296 \\
\hline 20 & 9.6593 & 12.2606 & 14.8011 & 17.3768 & 20.0470 & 22.8600 & 25.8640 & 29.1133 & 32.6744 & 36.6337 \\
\hline 21 & 9.6366 & 12.2122 & 14.7165 & 17.2436 & 19.8493 & 22.5775 & 25.4704 & 28.5738 & 31.9419 & 35.6425 \\
\hline 22 & 9.6162 & 12.1686 & 14.6409 & 17.1251 & 19.6744 & 22.3294 & 25.1275 & 28.1081 & 31.3163 & 34.8069 \\
\hline 23 & 9.5975 & 12.1286 & 14.5728 & 17.0189 & 19.5186 & 22.1097 & 24.8260 & 27.7018 & 30.7755 & 34.0924 \\
\hline 24 & 9.5805 & 12.0933 & 14.5112 & 16.9233 & 19.3790 & 21.9139 & 24.5588 & 27.3443 & 30.3034 & 33.4741 \\
\hline 25 & 9.5655 & 12.0606 & 14.4552 & 16.8368 & 19.2531 & 21.7382 & 24.3204 & 27.0271 & 29.8874 & 32.9336 \\
\hline 26 & 9.5506 & 12.0306 & 14.4040 & 16.7579 & 19.1390 & 21.5796 & 24.1063 & 26.7438 & 29.5180 & 32.4569 \\
\hline 27 & 9.5376 & 12.0031 & 14.3571 & 16.6859 & 19.0352 & 21.4358 & 23.9129 & 26.4892 & 29.1877 & 32.0333 \\
\hline 28 & 9.5253 & 11.9773 & 14.3140 & 16.6199 & 18.9402 & 21.3048 & 23.7374 & 26.2590 & 28.8906 & 31.6542 \\
\hline 29 & 9.5140 & 11.9540 & 14.2742 & 16.5591 & 18.8530 & 21.1850 & 23.5773 & 26.0499 & 28.6219 & 31.3129 \\
\hline 30 & 9.5033 & 11.9323 & 14.2373 & 16.5029 & 18.7728 & 21.0749 & 23.4308 & 25.8592 & 28.3776 & 31.0040 \\
\hline 31 & 9.4936 & 11.9119 & 14.2031 & 16.4509 & 18.6985 & 20.9734 & 23.2962 & 25.6845 & 28.1546 & 30.7230 \\
\hline 32 & 9.4845 & 11.8927 & 14.1712 & 16.4026 & 18.6297 & 20.8796 & 23.1720 & 25.5238 & 27.9502 & 30.4664 \\
\hline 33 & 9.4758 & 11.8753 & 14.1414 & 16.3576 & 18.5658 & 20.7926 & 23.0572 & 25.3755 & 27.7621 & 30.2309 \\
\hline 34 & 9.4677 & 11.8586 & 14.1136 & 16.3155 & 18.5062 & 20.7116 & 22.9506 & 25.2383 & 27.5885 & 30.0142 \\
\hline 35 & 9.4601 & 11.8430 & 14.0875 & 16.2762 & 18.4506 & 20.6362 & 22.8514 & 25.1109 & 27.4277 & 29.8140 \\
\hline 36 & 9.4528 & 11.8283 & 14.0629 & 16.2393 & 18.3984 & 20.5657 & 22.7589 & 24.9923 & 27.2784 & 29.6285 \\
\hline 37 & 9.4462 & 11.8145 & 14.0398 & 16.2046 & 18.3495 & 20.4996 & 22.6724 & 24.8817 & 27.1393 & 29.4561 \\
\hline 38 & 9.4393 & 11.8013 & 14.0181 & 16.1719 & 18.3036 & 20.4376 & 22.5914 & 24.7782 & 27.0094 & 29.2956 \\
\hline 39 & 9.4337 & 11.7889 & 13.9976 & 16.1411 & 18.2603 & 20.3793 & 22.5153 & 24.6811 & 26.8879 & 29.1456 \\
\hline 40 & 9.4279 & 11.7773 & 13.9779 & 16.1120 & 18.2194 & 20.3244 & 22.4437 & 24.5900 & 26.7740 & 29.0052 \\
\hline 45 & 9.4031 & 11.7269 & 13.8946 & 15.9877 & 18.0455 & 20.0914 & 22.1411 & 24.2063 & 26.2963 & 28.4191 \\
\hline 50 & 9.3833 & 11.6869 & 13.8290 & 15.8903 & 17.9098 & 19.9106 & 21.9075 & 23.9117 & 25.9318 & 27.9747 \\
\hline 55 & 9.3673 & 11.6546 & 13.7760 & 15.8118 & 17.8013 & 19.7662 & 21.7218 & 23.6785 & 25.6444 & 27.6260 \\
\hline 60 & 9.3538 & 11.6279 & 13.7322 & 15.7474 & 17.7119 & 19.6482 & 21.5706 & 23.4893 & 25.4120 & 27.3450 \\
\hline 65 & 9.3427 & 11.6054 & 13.6955 & 15.6935 & 17.6375 & 19.5500 & 21.4450 & 23.3326 & 25.2202 & 27.1137 \\
\hline 70 & 9.3330 & 11.5862 & 13.6643 & 15.6476 & 17.5745 & 19.4670 & 21.3391 & 23.2007 & 25.0591 & 26.9200 \\
\hline 75 & 9.3247 & 11.5697 & 13.6374 & 15.6082 & 17.5204 & 19.3958 & 21.2485 & 23.0882 & 24.9220 & 26.7554 \\
\hline 80 & 9.3175 & 11.5552 & 13.6140 & 15.5740 & 17.4735 & 19.3343 & 21.1702 & 22.9911 & 24.8038 & 26.6138 \\
\hline 85 & 9.3109 & 11.5424 & 13.5935 & 15.5440 & 17.4324 & 19.2804 & 21.1019 & 22.9064 & 24.7009 & 26.4907 \\
\hline 90 & 9.3054 & 11.5312 & 13.5753 & 15.5175 & 17.3961 & 19.2329 & 21.0416 & 22.8318 & 24.6104 & 26.3827 \\
\hline 95 & 9.3003 & 11.5213 & 13.5590 & 15.4939 & 17.3639 & 19.1907 & 20.9882 & 22.7658 & 24.5304 & 26.2871 \\
\hline 100 & 9.2958 & 11.5122 & 13.5445 & 15.4727 & 17.3350 & 19.1529 & 20.9404 & 22.7068 & 24.4589 & 26.2020 \\
\hline$\infty$ & 9.2103 & 11.3449 & 13.2767 & 15.0863 & 16.8119 & 18.4753 & 20.0902 & 21.6660 & 23.2093 & 24.7250 \\
\hline
\end{tabular}

